\title{
On Granular Stress Statistics: Compactivity, Angoricity, and Some Open Issues
}

\author{
Raphael Blumenfeld ${ }^{*},+*$ and Sam F. Edwards ${ }^{\dagger}$ \\ Biological and Systems, Cavendish Laboratory, J. J. Thomson Avenue, Cambridge CB3 OHE, U.K., and Earth \\ Science and Engineering, Imperial College, London SW7 2BP, U.K.
}

Received: November 5, 2008; Revised Manuscript Received: December 30, 2008

\begin{abstract}
We discuss the microstates of compressed granular matter in terms of two independent ensembles: one of volumes and another of boundary force moments. The former has been described in the literature and gives rise to the concept of compactivity: a scalar quantity that is the analogue of temperature in thermal systems. The latter ensemble gives rise to another analogue of the temperature: an angoricity tensor. We discuss averages under either of the ensembles and their relevance to experimental measurements. We also chart the transition from the microcanoncial to a canonical description for granular materials and show that one consequence of the traditional treatment is that the well-known exponential distribution of forces in granular systems subject to external forces is an immediate consequence of the canonical distribution, just as in the microcanonical description $E=H$ leads to $\exp (-H / k T)$. We also put this conclusion in the context of observations of nonexponential forms of decay. We then present a Boltzmann-equation and Fokker-Planck approaches to the problem of diffusion in dense granular systems. Our approach allows us to derive, under simplifying assumptions, an explicit relation between the diffusion constant and the value of the hitherto elusive compactivity. We follow with a discussion of several unresolved issues. One of these issues is that the lack of ergodicity prevents convenient translation between time and ensemble averages, and the problem is illustrated in the context of diffusion. Another issue is that it is unclear how to make use in the statistical formalism the emerging ability to exactly predict stress fields for given structures of granular systems.
\end{abstract}

\section{Introduction}

It has been shown ${ }^{1}$ that granular materials establish a reproducible state when shaken in a definite way, that is, by shaking at a given frequency and amplitude for a given length of time. This suggests that granular systems can be treated in a similar way to thermodynamic systems (via a Gibbsian discussion). The first question to ask is what macroscopic parameters characterize the steady state. The simplest thermal systems have energy $E$, volume $V$, and a number of particles $N$, in terms of which an entropy can be defined, $S(E, V, N)$. The temperature in such systems is defined as $T=\partial E / \partial S$. If an enclosed volume of granular material is shaken with a given frequency and amplitude for a long time, it will reach a volume $V$ to which it can return after a disturbance and reshaking. If this volume is enclosed by a surface, which can sustain a loading stress, and is again excited repeatedly in a similar manner, then its properties will also eventually reach steady state values. The number of configurations of the granular material is astronomically large, and an entropy can be defined, $S(V, N)$. The Lagrange multiplier, which is analogous to the temperature, can be obtained, for example, by the standard Gibbs passage from the microcanonical to the canonical description, giving the compactivity $X=\partial V / \partial S .^{2}$

Although both the temperature and the compactivity are scalars, this need not be the general case. It is illustrative to recall that, when angular momentum $\Omega$ is a relevant variable in conventional thermal systems, the entropy is $S(E, \omega, N, V)$ and, in addition to the traditional temperature, there appears a second tensorial temperature, $\tau_{i j}=\partial \Omega_{i j} / \partial S^{3}$ The distribution

\footnotetext{
* To whom correspondence should be addressed. E-mail: rbb11@ cam.ac.uk.

${ }^{\dagger}$ Cavendish Laboratory.

* Imperial College.
}

function $\exp (F-H / k T)$ is then extended to $\exp (\tilde{F}-H /(\partial E /$ $\partial S)-\Omega /(\partial \omega / \partial S))$, where $\omega$ is the expectation value of the operator $\Omega$ just as $E$ is the expectation value of the operator $H$.

We must therefore ask what quantities characterize powder that has reached a terminal state. The simplest case to consider is of a powder inside a membrane, immersed in a liquid that exerts a pressure $P$. Then the grains will have an orientation, a position, contacts with other grains, and a distribution of forces at these contacts. Thus there will be contacts $\vec{r}^{\alpha \beta}$ with centroids $\vec{R}^{\alpha}=(1) /\left(z^{\alpha}\right) \Sigma_{\beta} \vec{r}^{\alpha \beta}$, and forces $\vec{f}^{\alpha \beta}$ giving force moments $\mathscr{F}^{\alpha}$ $=\Sigma_{\beta} \vec{f}^{\alpha \beta} \otimes \vec{r}^{\alpha \beta}$. The set of $\vec{r}^{\alpha \beta}$ and $\vec{f}^{\alpha \beta}$ specify a configuration of the powder.

When the powder is shaken these quantities change, but after repeated shaking the grains will fill a volume $V$ and exert a stress $\hat{\Sigma}$ on the boundary. It is presumed that the repeated shaking moves the system between typical configurations in the configuration phase space, which are subject to the constraints on $V$ and $\hat{\Sigma}$. The system must then have an entropy $S(V, \hat{\Sigma})$ and allows the definition of temperature-like quantities $\partial S / \partial \Sigma_{i j}$ and $\partial S / \partial V$. Just as $\delta(E-H)$ defines the microcanonical ensemble and gives rise to the canonical form $\exp [F-H /(\partial E / \partial S)]$, there should be an analogue of $H$ that is a volume function $W$, and the microcanonical description is $\delta(V-W)$. Including the effects of the external loading on the surface via $\delta^{d(d+1) / 2}\left(V \Sigma_{i j}-\Phi_{i j}\right)$ gives

$$
\mathrm{e}^{-S} \delta(V-W) \delta\left(V \Sigma_{i j}-\Phi_{i j}\right)
$$

where $\Phi_{i j}$ is the $i j$ th component of a force moment tensor function, 


$$
\hat{\Phi}=\sum_{\alpha, \beta} \vec{r}^{\alpha \beta} \otimes \vec{f}^{\alpha \beta}
$$

where $d$ is the dimension, and $d(d+1) / 2$ is the number of independent components of the force moment. To make progress, we assume for simplicity that granular systems can be described sufficiently well by a flat measure. Passing to the canonical distribution in the standard Gibbs fashion, as in any standard textbook, then gives

$$
\mathrm{e}^{F-W /(\partial V / \partial S)-\Phi_{i j} /\left(\partial\left(V \Sigma_{i j}\right) / \partial S\right)}
$$

We seek one local volume function $V^{q}$ such that the total volume is

$$
V=\sum_{q} V^{q}
$$

where $q$ is a basic volume element with which we can fill the space occupied by the granular system. The filling (tessellation) method, must correspond uniquely to the connectivity of the granular system, and it has been developed for two-dimensional systems in refs 6 and 7 and for three-dimensional systems in refs 8 and 10. Describing the shape of a basic volume element $q$ by a fabric tensor, $\hat{C}^{q}$, as defined below, the volume function can be obtained from the trace of the tensor both in two and in three dimensions:

$$
V^{q}=\frac{1}{d} \operatorname{Tr}\left(\hat{C}^{q}\right)
$$

In two dimensions the fabric tensor is defined as the outer product

$$
\hat{C}^{q}=\hat{\varepsilon} \cdot \vec{r}^{q} \otimes \vec{R}^{q}
$$

where $\hat{\varepsilon}$ is the $2 \times 2$ Levi-Civita tensor and the vectors $\vec{r}^{q}$ and $\vec{R}^{q}$ are the diagonals of the quadrilateral $q$ shown in Figure 1a. ${ }^{6,7,9}$ These elements have been named quadrons. ${ }^{7}$ Every grain is made of $z$ quadrons, where $z$ is the grain's number of contacts (coordination number).

In three dimensions the tensor is

$$
\hat{C}^{q}=\left(\xi^{q} \times \vec{r}^{q}\right) \otimes \vec{R}^{q}
$$

where the construction of the space-filling quadrons and the corresponding vectors $\xi^{q}, \vec{r}^{q}$, and $\vec{R}^{q}$ are shown in refs $8-10$.

By summing over quadrons that extend between the centroid of a specific grain and the centroids of the cells that surround it, we get the volume associated with the grain, both in $2 \mathrm{D}$ and in $3 \mathrm{D}$. This is illustrated in the $2 \mathrm{D}$ example in Figure 1. These grain volumes also sum up to the total volume of the system. Therefore, at first glance, the volume function may be obtained either as a sum over those grain volumes or over the basic quadron volumesm, where the correct volume function depends on the number of degrees of freedom in the system. It has been shown in ref 7 that in $2 \mathrm{D}$ the number of degrees of freedom is the same as the number of quadrons. In three-dimensional systems of mean coordination number $z=4$ (the marginal rigidity value for grains with high friction coefficients), the
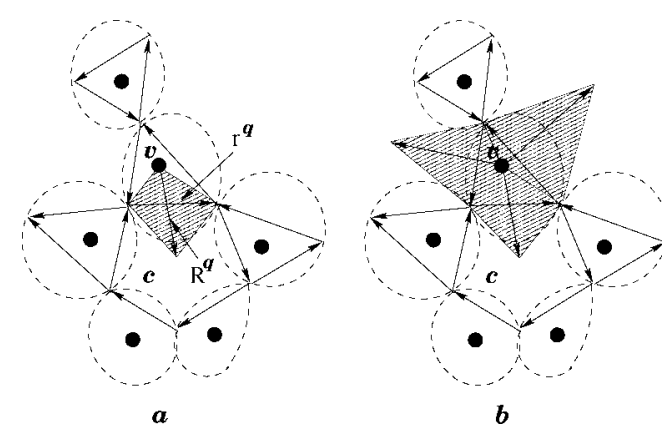

Figure 1. The geometric construction around grain $v$ in 2D. The vectors $\vec{r}^{q}$ connect contact points anticlockwise around each grain (and clockwise around each cell c). The vectors $\vec{R}^{q}$ connect from grain centroids to centroids of neighboring cells. (a) The quadrilateral, or quadron, associated with grain $v$ and cell $c$ is shown shaded. (b) The sum of the areas of all the quadrons around grain $v$ constitute the area associated with this grain. The sum of all these areas is the area of the entire system.
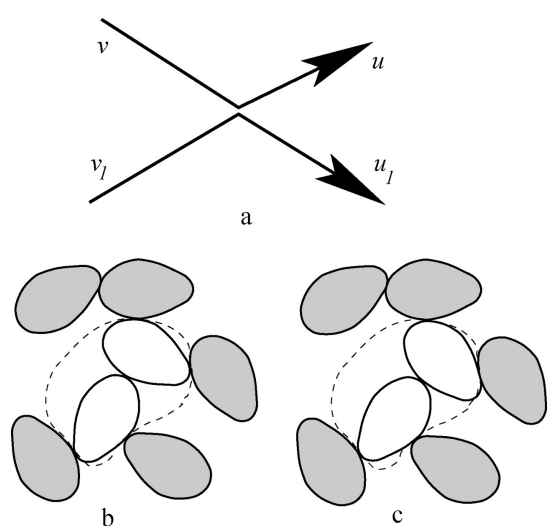

Figure 2. (a) Boltzmann's approximation of two-particle scattering and losing memory of the collision before ever colliding again; (b) part of a multicontact system of grains; (c) the same system of grains slightly evolved at a later time; note the continued contacts during the evolution of the central grains.

number of degrees of freedom per grain is much smaller than the number of quadrons. ${ }^{8,10}$ This already suggests that simply summing in the volume function over grains is inaccurate. Indeed, a recent analysis has shown that using the quadrons as the basic "quasiparticles" of the statistical mechanical formalism leads to a better insight than using grains into the role of grain characteristics in forming the structure of a granular system. ${ }^{11}$

The choice of the identities of the basic quasiparticles in the partition function also determines the ensemble to use. When using quadrons as quasiparticles, one is required to use a grand canonical ensemble. The reason is that the number of contacts between particles may change from configuration to configuration even if the number of particles does not. In isostatic states, where the mean coordination number is fixed, the change in the number of contacts may be negligible, being caused only by boundary effects. In such cases, taking the system size to infinity makes it possible to again use the canonical ensemble, even for a system of quadrons; but in ensembles that contain nonisostatic states, when the mean coordination number is not constrained, only the grand canonical ensemble will do. Indeed, it is likely that this latter case is more relevant for many experimental set-ups because in most reported experiments the number of particles is normally fixed, but there is little, or no, control over the number of contacts. In particular, it is also likely that such was the situation in the seminal experiment carried out by the Chicago group. ${ }^{1}$ We therefore urge experimentalists 
to keep track of the coordination numbers; this is probably one of the most significant microscopic parameters that determine the state of a granular system.

Given the number of ways that the grains can occupy the volume $V$ and support the boundary stresses $\hat{\Sigma}$, one can define an entropy $S(V, N, \hat{\Sigma})$. It is plausible that a given structural configuration of grains can support a range of configurations of boundary loads. Correspondingly, it is also plausible that, given a specific macroscopic boundary stresses, there is a range of structural configurations that are stable under it. This leads to the idea that, in addition to the ensemble of geometric configurations at a given volume, we should consider an independent ensemble of the boundary force configurations that gives rise to $\hat{\sigma}$. Following a similar analysis as the one leading to the concept of the compactivity, there should be a temperature-like Lagrange multiplier that is associated with the force ensemble. However, since the stress is a tensor, then so should be that particular temperature-like quantity. This is no different than in conventional thermal systems in the presence of angular momentum $\Omega$, as described above. Thus, in granular system there are two analogues of temperature: the scalar compactivity $X=\partial V / \partial S$ and the (tensorial) angoricity $Z_{i j}=\partial \hat{\Sigma}_{i j} / \partial S .^{12-14}$ The latter may be simplified for systems loaded by a pure hydrostatic pressure $P$, in which case the angoricity degenerates to $Z_{0}=$ $\partial P / \partial S$.

The partition function is then

$$
\int D(q) \mathrm{e}^{-W(q) / X-\Phi_{i j}(q) / Z_{i j}} \Theta(q)
$$

where the degrees of freedom now include, in addition to the geometric degrees of freedom discussed above, the forces on the boundary grains; and the function $\Theta$ contains $\delta$-functions that ensure mechanical equilibrium. In the simplified case, when the system is under a hydrostatic pressure, the distribution of structural and force configurations is given by eq 9 .

$$
\mathrm{e}^{F-W(q) / X-P(q) / Z_{0}}
$$

Indeed, it has been discovered some time ago that the distribution of the magnitudes of the intergranular forces in granular systems has an exponential tail, ${ }^{15}$ which has been followed by a number of microscopic models to explain it. ${ }^{16}$ However, the above analysis suggests that this feature might be very general and that it is simply a consequence of the canonical distribution. Nevertheless, this issue is not entirely resolved, as we will discuss briefly later. In particular, experiments that find deviations from exponential tails ${ }^{24}$ may suggest that some of the assumptions made here may not be valid in particular situations.

\section{The Boltzmann Equation}

A little addressed question in the literature has been whether the insight from the statistical-mechanical approach can be extended to understand dynamics of granular systems in the same way that the traditional approach can be used to construct the Boltzmann and the Fokker-Planck equations. In this section we turn to discuss an attempt in this direction for granular systems.

The classic Boltzmann equation studies dilute gases whose dynamic evolution presumes two-body collisions. Thus, assuming that our gas is described by a probability $f(\vec{r}, \vec{v}, t)$ of finding a molecule at $\vec{r}$ with $\vec{v}$ at the time $t, f$ will evolve subject to the equation

$$
\begin{aligned}
\frac{\partial f}{\partial t}+\vec{v} \cdot \frac{\partial f}{\partial \vec{r}}+\int K\left(\vec{v}, \vec{v}_{1}, \vec{u}, \vec{u}_{1}\right)\left[f(\vec{r}, \vec{v}, t) f\left(\vec{r}, \vec{v}_{1}, t\right)+\right. \\
\left.\frac{\vec{F}}{m} \cdot \frac{\partial f}{\partial \vec{v}}-f(\vec{r}, \vec{u}, t) f\left(\vec{r}, \vec{u}_{1}, t\right)\right] d^{3} \vec{v}_{1} d^{3} \vec{u}^{3} \vec{u}_{1}=0
\end{aligned}
$$

This equation contains a scattering kernel $K$, representing a particle with $\vec{v}$ at $\vec{r}$ and $t$ meeting another with $\vec{v}_{1}$ at the same $\vec{r}$ and $t$, in which case they scatter, respectively, into new velocities $\vec{u}$ and $\vec{u}_{1}$. The last term represents the change in the distribution function due to a change in the external forces $\vec{F}$, or boundary conditions. This equation successfully describes simple transport such as the viscosity and the thermal conductivity of gases. Boltzmann showed that, if the entropy of the gas is defined by $S=-\int f \ln f$, then $\partial S / \partial t \geq 0$. Also, since $K$ ensures the conservation of energy and momentum then $f(\vec{r}, \vec{v}, t) f\left(\vec{r}, \vec{v}_{1}, t\right)$ $=f(\vec{r}, \vec{u}, t) f\left(\vec{r}, \vec{u}_{1}, t\right)$ provided that $f=n e^{\left.-m|\vec{v}-\vec{v}|^{2} / K_{B} T\right)}$, where $n$ is the density, and $\overline{\vec{v}}$ is the mean velocity.

The question is how to extend this to granular systems? The Boltzmann equation above has been used extensively for granular gases, sometimes by using the extension of the equation by Enskog to dense systems. For real dense granular systems, this approximation fails miserably; grains are either in permanent contact with neighbors or exchange contacts frequently. The typical classical picture of two particles scattering off one another (Figure 2a) is substantially different than the multicontact picture in granular systems (Figures $2 b-c$, drawn in $2 \mathrm{D}$ for simplicity).

In $\dot{d}$-dimensional static systems of frictional particles in mechanical equilibrium, one expects at least $z_{\mathrm{c}}=d+1$ contacts. Experimentally, by minimizing inertial effects when generating granular packs, the mean number of contacts can be very close to this value. ${ }^{17}$ However, under more violent processing procedures, or by changing pack-generation protocols, the mean number of contacts can be made higher. Nevertheless, for the system to be dynamic, the mean number of contacts must drop sufficiently close to $z_{\mathrm{c}}$, at least for hard (but not infinitely hard, see e.g., ref 18) particles. A key point is that in dense systems every grain is in contact most of the time, for example, the transition from Figure $2 \mathrm{~b}$ to $2 \mathrm{c}$ has the grains continually in contact. This is true even if contacts are broken and new ones are made, which is obviously the case in a dense flow situation.

Let us consider the simplest situation, when each particle maintains persistently exactly four contacts during the change in configuration. In this case, the granular analogy of eq 10 takes the form

$$
\begin{array}{r}
\frac{\partial f}{\partial t}+\int K\left[f \Pi_{i=1}^{4} f_{i}-f^{\prime} \Pi_{i=1}^{4} f_{i}^{\prime}\right] d\{\text { all deg. of freedom }\} \\
+ \\
\text { (forces) }=0
\end{array}
$$

where $f_{i}\left(f^{\prime}{ }_{i}\right)$ is the distribution of grain $i$ before (after) the "collision" event (we use this conventional language to make contact with traditional analyses, although in our dense system this is not a discrete event but rather a continuous evolution, on top of which there are topological changes). For the diluted gas, $f$ depends on $\vec{v}, \vec{r}$, and $t$. What can it depend on in a dense granular material? Ideally, we should seek variables that either label a single grain or a quadron. As mentioned above, the two lead to completely different approaches. Quadrons, which are more convenient for static ensembles, are structural constructs whose identities are not conserved. Quadrons are created and annilhilated as contacts are made and broken. This is no different than systems of photons or phonons. However, this makes the 
treatment more involved, and we will discuss this elsewhere. Here we choose the grains as the basic particles, with the convenience that their identities and number are conserved. We can use the position of a grain $\vec{R}^{\alpha}$ and its fabric tensor $C^{\alpha}$ as descriptors of its status of the grain relative to its neighbors. Although this is a very approximate picture, it nevertheless yields interesting results. The change of positions of the central grains in Figure 2 change not only the volume associated with them but also those associated with the grains surrounding the cells immediately adjacent to them. The smallest number of grains involved in a change of volume of one grains is four. The kernel $K$ conserves volume and stress by choice of the ensemble that we study (see discussion below), so that for the smallest possible change of volumes

$$
V^{\alpha}+\sum_{j} V^{j}=U^{\alpha}+\sum_{j} U^{j}
$$

So just as conservation of momentum and energy in conventional systems,

$$
\sum_{k} \vec{v}_{k}=\sum_{k} \vec{u}_{k} \text { and } \sum_{k} v_{k}^{2}=\sum_{k} u_{k}^{2}
$$

where $k$ runs over the particles involved in the collision, leading to a distribution of a Gaussian form

$$
\mathrm{e}^{-\frac{1}{2}(\vec{v}-\overline{\vec{v}})^{2} / k_{\mathrm{B}} T}
$$

and the conservation of volume gives

$$
\mathrm{e}^{-W / X}
$$

where $X=\partial V / \partial S$.

Likewise, the force moments resolve the stress transmission and

$$
\mathscr{F}+\sum_{k} \mathscr{F}_{k}=\widetilde{\mathscr{F}}+\sum_{k} \widetilde{\mathscr{F}}_{k}
$$

where $\mathscr{T}_{k}$ is the force moment of the $k$ th grain after the change in configuration, and this gives the distribution

$$
\mathrm{e}^{-F_{i j} / Z_{i j}}
$$

where

$$
Z_{i j}=\frac{\partial F_{i j}}{\partial S}
$$

is the component of the temperature-like angoricity tensor, discussed earlier.

We consider an ensemble of all possible systems with a given volume $V$ and boundary stresses $\sigma_{i j}$. We divide this ensemble into two subensembles. In one, which we name P-ensemble, the boundary loads are kept constant, in the sense that the force load on every boundary particle is fixed. This ensemble consists of all possible structural configurations of the particles in mechanical equilibrium whose total volume is $V$. The other subensemble, named $\mathrm{V}$-ensemble, consists of all the possible realizations of boundary forces that add up to a total boundary stress $\sigma_{i j}$ for a fixed particular configuration of the particles in the system. It is significant to realize that the two ensembles are independent. This is in contrast to the assumption made in the literature that every combination of the load forces on the boundary can have only one structural configuration of the system that corresponds to it. ${ }^{21}$ To understand this, consider an arbitrary system of grains in mechanical equilibrium under a particular set of boundary forces. By boundary forces we mean here the total stress on the boundary, not a configuration of forces on each and every boundary particle. The material has an yield stress, and let us assume that the stress state is such that the material is not exactly on the yield surface. Therefore, the stress on the boundary of the system can be changed without any of the particles moving. It follows that, while the structure of the system has not changed, the boundary stress has. This argument shows that we can have a range of boundary stress values for a particular given structural configurations and that the two ensembles are independent, that is, changing one does not necessarily result in the change of the other.

So one recovers the "Gibbs" solution from the Boltzmann equation, but the kernel function $K$, which describes the transition between states $b$ and $c$ in Figure 2 (which also includes constraints on this transition), involves diabolical algebra, albeit of a 19th century vintage. Moreover, Boltzmann's $H$ theorem, that entropy defined by $-\int f \ln f D\{q\}$ always increases with time, also follows for granular systems satisfying the above conditions. $^{22}$

In conventional statistical mechanics it is possible to expand, for soft or long-range forces, in the change of momentum, namely, in $\vec{v}-\vec{u}$ and $\vec{v}_{1}-\vec{u}_{1}$, which turns Boltzmann's integral equation into a differential form: the Fokker-Planck equation. Next, we try this approach for granular media.

\section{The Fokker-Planck Equation}

To make progress we require a simple equation, which can only arise from employing one-particle variables. For example, in the classic Boltzmann equation these are the position and momenta of any given particle. For a grain, a straightforward position variable is $\vec{R}^{\alpha}$, but this is not a true single variable since it depends on the contacts, which in turn depend on positions of neighboring grains. Nevertheless, for grains of aspect ratios around 1 , the error due to changes of contacts cannot be too large. The volume associated with grain $\alpha$ is determined from the fabric tensor. This suggests that, if we restrict the discussion for the moment to position and volume, we should consider a distribution function that depends on the position vector $\vec{R}$, the fabric tensor $\hat{C}$ and time $t, f(\vec{R}, \hat{C}, t)$. Since the volume can be calculated from the fabric tensor, we write it formally in the following as $V(\hat{C})$.

In conventional systems, the Fokker-Planck equation can be derived from the Boltzmann equation by considering the case of weak scattering and expanding as mentioned above, which leads to the equation

$$
\frac{\partial f}{\partial t}+\vec{v} \cdot \frac{\partial f}{\partial \vec{r}}+\frac{\partial}{\partial \vec{v}} \cdot\left[\hat{D} \cdot \frac{\partial}{\partial \vec{v}}+\hat{\mu} \cdot \vec{v}\right] f=0
$$

where in equilibrium the tensors $\hat{D}$ and $\hat{\mu}$ are related by the fluctuation-dissipation theorem. The Fokker-Planck equation extends beyond equilibrium and is covered well in a paper by 
Chandrasekhar. Out of equilibrium, $\hat{D}$ is the diffusivity and $\hat{\mu}$ is the dynamic friction.

There is a wide literature on the application of the Boltzmann equation to dilute granular systems, ${ }^{20}$ but these works are not relevant to the systems that we are interested in here. The main difference can be traced to the mean number of contacts between grains, $z$. While in the dilute limit, this number is close to zero, we consider only systems that are either jammed or close to jamming. The application of the Boltzmann equation to such systems has hardly been explored (but see ref 21 ). If we can consider solely the case of packing, that is, $\hat{D}=0$, then $f(\vec{R}, \hat{C}, t)$ $=\mathrm{e}^{-V(\hat{C}) / X}$, and it follows that the Fokker-Planck equation, appropriate to the steady state, but which covers fluctuations, is

$$
\frac{\partial f}{\partial t}-\sum_{i j k l} \Lambda_{i j k l}\left(\frac{\partial}{\partial C_{k l}}+\frac{\bar{z}}{\mathrm{~d} X} \delta_{k l}\right) f=0
$$

where $\Lambda$ depends on the shaking mechanism and does not affect the form of the steady state distribution, $\bar{z}$ is the mean coordination number, and the last term on the left-hand side follows from eq 5 .

A simple approximation is to replace $\Lambda$ by its average, leading to $\Lambda_{i j}=\Lambda(X) \delta_{i j} \delta_{k l}$, and treat $W$ as the volume variable. This leads to

$$
\frac{\partial}{\partial t}-\frac{\partial}{\partial W} \Lambda\left(\frac{\partial}{\partial W}+1 / X\right) f=0
$$

whose solution is $f=\exp (-W / X) / X$. This is a simplified calculation, and a fuller description involves nonconstant Jacobians, which we will not go into in this presentation.

At this level we can introduce $P$ as a variable too. Then $f=$ $f(X, Z, W, P, t)$ depends on the compactivity $X$, the angoricity $Z$, the fabric tensor $P$, and time $t$, and it must satisfy

$$
\left[\frac{\partial}{\partial t}-\frac{\partial}{\partial W} \Lambda_{1}\left(\frac{\partial}{\partial W}+\frac{1}{X}\right)-\frac{\partial}{\partial P} \Lambda_{2}\left(\frac{\partial}{\partial P}+\frac{1}{Z}\right)\right] f=0
$$

The question one now faces is the extent of detail needed to employ to resolve granular problems. The existence of equations and of an $\mathrm{H}$-theorem, which can be used to address and obtain quantitative solutions to some simple problems, is satisfactory, but it is far from sufficient to understand the richness of granular behavior, and a range of complex issues remain unresolved.

It is worth working out a crude version in order to obtain a solution for a particular physical systems. The compactivity $X$ is at its lowest (highest) when the system is as closely (loosely) packed as possible. Thus, the Fokker-Planck equation for the random movement of grains due to random forces acting on them takes the form

$$
\left(\frac{\partial}{\partial t}+\vec{F} \cdot \frac{\partial}{\partial \vec{r}}\right) \phi=0
$$

where $\vec{F}$ is a random force field, whose distribution is given by $\mathrm{e}^{-\int|\vec{F}|^{2} \mathrm{~d} t / A}$. This leads, traditionally, to

$$
\left(\frac{\partial}{\partial t}-A \frac{\partial^{2}}{\partial r^{2}}\right) G\left(r, r^{\prime}, t, t^{\prime}\right)=\delta\left(r-r^{\prime}\right) \delta\left(t-t^{\prime}\right)
$$

However, when our nonlinear terms are present, this becomes

$$
\left[\frac{\partial}{\partial t}-A \frac{\partial}{\partial r}\left(\frac{\partial}{\partial r}+\frac{1}{X} \frac{\partial W}{\partial r}\right)\right] G\left(r, r^{\prime}, t, t^{\prime}\right)=\delta\left(r-r^{\prime}\right) \delta\left(t-t^{\prime}\right)
$$

in order that the steady state has the probability $\mathrm{e}^{-W / X}$.

Both $W$ and $X$ have dimensions of volume, and the fundamental volume in the system is that associated with a typical grain, which we denote as $a^{3}$. Thus, an approximate mean field type of the model could be

$$
\begin{aligned}
& {\left[\frac{\partial}{\partial t}-A \frac{\partial}{\partial r}\left(\frac{\partial}{\partial r}+\frac{\left.\left(r-r^{\prime}\right)\right)^{2}}{X}\right)\right] G\left(r, r^{\prime}, t, t^{\prime}\right) }= \\
& \delta\left(r-r^{\prime}\right) \delta\left(t-t^{\prime}\right)
\end{aligned}
$$

Considering the correlation function

$$
Q \equiv\left\langle\left(r-r^{\prime}\right)^{2}\right\rangle
$$

its rate of change from state to state is

$$
\frac{\partial Q}{\partial t}=2 A\left(1-\frac{2 a^{3}}{X}\right)
$$

Note that the concept of time in this expression is somewhat artificial. It should be regarded as a parameter that quantifies the change from configuration to configuration. The reason that we cannot use time in the conventional sense has to do with the lack of ergodicity, as will be discussed briefly in the concluding section. We note that when $X=\infty$ the system (which should still be mechanically stable under some specified mechanical loading) is at its loosest possible state (might as well be the loose random packing under these particular conditions). This identifies the meaning of the coefficient $2 A$; it is the rate of change of $Q$ at this state. In the state when the compactivity is lowest, $X=2 a^{3}, Q$ does not change, $\partial Q / \partial t=$ 0 , corresponding to states that are sufficiently compact to freeze any possible movement of grains. Since this is the smallest possible compactivity, it is convenient to redefine the compactivity such that it vanishes at the frozen state. Then, in terms of the new compactivity,

$$
\frac{\partial Q}{\partial t}=2 A\left(1-\frac{1}{X / 2 a^{3}+1}\right)
$$

This expression also shows that it is natural to measure any volume in units of the typical volume associated with one grain. This is of course true as long as such a value is a good descriptor, namely, when the particle size distribution is not heavy-tailed. Thus, rescaling all the volumes by $2 a^{3}$, we have

$$
\frac{\partial Q}{\partial t}=2 A\left(1-\frac{1}{X+1}\right)
$$

and

$$
Q=\frac{2 A X}{X+1} t
$$


It is emphasized that the above is a very crude approximation, offered here only to illustrate a way to approach the "diffusion" problem. Nevertheless, eq 31 is quite interesting in that it offers a way of relating the diffusion coefficient to the compactivity. The latter has been hitherto an elusive and somewhat abstract quantity, whereas the former is readily accessible to numerical and experimental measurements. ${ }^{23} \mathrm{We}$ expect that, as the compacitivity $X \rightarrow 0$ the system densifies and approaches a random close packing, in which case the diffusivity should vanish. In contrast, when the compactivity gets very high, $X \rightarrow$ $\infty$, the system is connected as loosely as possible (a marginally rigid state), ${ }^{17}$ and the diffusion coefficient is simply $A$. If we take, for example, the value for the diffusion coefficient, found by Utter and Behringer in ref 23 (read from the slope of the tangential diffusion in their Figure 4$), Q\left(X_{\mathrm{UB}}\right)=2 D_{\mathrm{UB}} \approx 0.02$, then the compactivity of their experiment is

$$
X_{\mathrm{UB}}=\frac{1}{1-Q(\infty) / 0.02}
$$

\section{Open Issues}

In the above we discussed only several aspects that relate to the statistics of granular materials. There are many more facets to the science of these systems, and there is a number of open issues that hinder progress in the field. Arguably, the most fundamental are the lack of full continuum theories for (i) the manner in which granular materials transmit static stresses and (ii) for their rheology in the dense state. Much effort is invested in attacking these problems, and we do not presume to make progress on them here per se. Rather, our aim in the following is to draw attention to open issues, highlighted by the recent developments in the statistical description presented above.

A. Nonergodicity and the "Diffusion" Process. One of the most significant consequences of ergodicity in thermal physics is that the statistics of an ensemble of appropriately equilibrated systems at different states is the same as those of snapshots of one system at different times. For example, carrying out conventional measurement on $B(\gg 1)$ same-volume boxes of air at room temperature should give the same results as carrying out the same experiments on one of the boxes at $B$ different times. This is a powerful assumption that allows us to obtain theoretical results in whatever statistics that are most amenable. This assumption works well in the case of the air in the box because during one measurement the air molecules undergo so many collisions and changes of state that the limit statistics always remain a good description. Unfortunately, because of the sluggish dynamics of granular systems, to which thermal fluctuations are hardly relevant, there is no such rapid transition between states, and during realistic experimental measurement on human time scales steady-state statistics are not easily established. As a result, we have no access to the powerful simplification offered by ergodicity.

Let us consider again the Chicago experiment. A volume of grains is shaken for some time, following a particular protocol, and then brought to rest. That experiment established that doing this leads to a steady-state distribution of a particular quantity, the density, in the final rest states. It is probably safe then to assume that shaking by the same protocol many times will always result in statistically similar final states and, therefore, that the ensemble of final states is a typical representation of the steady-state statistics. It is important to remember that the statistics does not describe in any way the dynamical states between the static final states.
Now suppose that in the experiment one inserts into the shake system a tracer particle, whose position can be detected at any time (and possibly even the velocity during the dynamic phase between final states). Suppose that the experiment is repeated many times. Let the rest states between shakes be labeled in order of occurrence $S_{k}$, with $n=1,2, \ldots, N$ and let us assume that we monitor the tracer's location at every such state, $\vec{r}_{n}$. Essentially, the integer $n$, which labels the sequence of states, is the analogue of time, and it is the parameter $t$ in eqs $28-31$.

This type of experiment has been carried out under different protocols by a number of groups, often with the aim to understand the dynamics of the tracer and relate the dynamics to diffusivity. However, is such a relation simple? In thermal systems one can analyze a dynamic model and relate it to the ensemble statistics by relying on ergodicity. The problem with the experiments just described is that we cannot approximate the change in the position of the tracer from final state to final state by a kinematic approach. All we can observe is a stroboscopic sequence of snapshots with a black-box type of dynamics leading from one to the next.

Moreover, if the shaking is sufficiently long and effective so as to make absolutely certain that the limiting distribution has been reached, as presumably was the intent in the original Chicago experiment, then there should be no correlation between sequential positions of the tracer. This throws even more confusion on any attempt to understand this diffusion process.

B. The Tail of the Intergranular Force Distribution: Exponential or Gaussian? Another interesting issue concerns the attempts to understand intergranular force distributions directly from the statistical approach, for example, as described above. In particular, it is still not clear whether the tail of the force distribution is indeed exponential or not. Statistics depend sensitively on the level of knowledge of the system. If only the total volume and the global force moments are known, then it is straightforward to deduce that the tail of the distribution must be exponential.

Some experimental ${ }^{24}$ and numerical ${ }^{25}$ studies have found deviations from an exponential form. Such observations suggest that the assumptions used in the derivations mentioned above may rely on inaccurate assumptions. Indeed, it has been proposed that there may be another constraint on the system: a conservation of the total area of a reciprocal tiling, which is a consequence of local force balance. ${ }^{26}$ If this claim is established, it could lead to the conclusion that the tail of the force distribution may in fact be Gaussian.

C. Effects of Stress Solutions. The statistics may be further affected by additional knowledge of another source: the general knowledge of the stress solutions. Much effort in the community has been invested in an attempt to understand stress transmission in quenched granular systems, namely, in systems whose structures are given. Applying external loads to these structures, the problem addressed in these studies has been the prediction of the stresses and forces that develop. The list of works on this problem is far too long to cite it here.

This problem has also been the source of a fierce debate in the community concerning the continuous stress theory that describes such stress solutions in granular materials. Until recently, the main candidates have been elasticity, plastoelasticity, and isostaticity theories. Recently, it has been suggested that for the purpose of understanding stress transmission, granular media should be regarded as two-phase composites; one phase being isostatic and the other consisting of overconnected regions, whose mean contact number per grain exceeds the marginal rigidity value, $z_{\mathrm{c}}$. The suggestion then was that 
isostaticity theory applies in the former, whereas in overconnected regions either elasticity or plaso-elasticity apply. ${ }^{9}$ Whichever is the correct theory, it should predict in principle the exact general solutions for given loading data and constitutive properties (i.e., elastic parameters in the case of elasticity and structural descriptors in the case of isostaticity). Thus, if we know the statistics of the constitutive parameters, whichever those are, and the loading data, then we should also be able to know the stress states and therefore also the statistics of the stress field. Yet, there seems to be no provision for including this knowledge in the statistical approach currently discussed in the literature.

To illustrate the point, consider a purely isostatic granular media. The stresses in such materials are described by isostaticity theory, for which the constitutive properties are structural. Specifically, they consist of the local values of a particular fabric tensor, $\hat{P}=1 / 2\left(\hat{C}+\hat{C}^{T}\right)$, where $\hat{C}$ has been described above. The fabric tensor is only a function of the vectors that make the contact network (more precisely, a subset of independent such vectors). Given a particular structure, it is then possible to construct the fabric tensor everywhere ${ }^{6}$ and, under specified boundary loading, solve for the stress field ${ }^{19}$ explicitly. Therefore, if we knew the statistics of the degrees of freedom for all possible structural configurations (which is equivalent to knowing the density of states in the volume ensemble) then we could know in principle the statistics of the stress solutions.

Yet, the entropic formalism, based on the volume ensemble (compactivity) and the boundary forces ensembles (angoricity) does not take at all into consideration the fact that these solutions are known.

One way to remedy this situation is by introducing constraints on the stress field in the partition function. This, however, is a project that has not been taken yet by anyone, and it is the opinion of these authors that this issue requires more discussion and work.

\section{References and Notes}

(1) (a) Nowak, E. R.; Knight, J. B.; Ben-Naim, E.; Jaeger, H. M.; Nagel, S. R. Phys. Rev. 1998, E 57, 1971. (b) Philippe, P.; Bideau, D. Europhys. Lett. 2002, 60, 677.

(2) (a) Edwards, S. F. Oakeshott, R. B. Physica 1989, D 38, 88. (b) Physica A 1989, 157, 1080. (c) Mehta, A.; Edwards, S. F. Physica 1989, A 157, 1091. (d) Edwards, S. F. In Proceedings of the International School of Physics: Enrico Fermi; Chiarotti, G. E., FumiE., TosiM. P. Eds.; North Holland, NY 1990; Vol. 106. 1958

(3) Landau L. D.; Lifshitz, E. M. Statistical Physics; Pergammon Press,

(4) Mehta, A.; Edwards, S. F. Physica 1989, A 157, 1091.
(5) (a) Edwards, S. F.; Grinev, D. V. Phys. Rev. 1998, E 58, 4758. (b) Chem. Eng. Sci., 2001, 56, 5451.

(6) Ball, R. C.; Blumenfeld, R. Phys. Rev. Lett. 2002, 88, 115505.

(7) Blumenfeld, R.; Edwards, S. F. Phys. Rev. Lett. 2003, 90, $114303-$ 114306.

(8) Blumenfeld, R.; Edwards, S. F. Eur. Phys. J. 2006, E 19, 23-30.

(9) Blumenfeld, R. Phys. Rev. Lett. 2004, 93, 108301.

(10) Blumenfeld, R. In Lecture Notes in Complex Systems; Aste, T. Tordesillas, A., Matteo, T. D.; World Scientific: Singapore 2008; pp 4353.

(11) Frenkel, G.; Blumenfeld, R.; Zdenek, G.; King, P. R. Phys. Rev. 2008, E 77, 041304 .

(12) Edwards, S. F. In Powders and Grains, Stuttgart; Garcia-Rojo, R. Herrmann, H. J., McNamara, S. Eds.; Balkema: Leiden, Netherlands, 2005; pp 3-5.

(13) (a) Edwards, a. S. F.; Blumenfeld, R. In Granular Physics; Mehta, A. Ed.; Cambridge University Press: Cambridge, 2007; pp 209-232. (b) Snoeijer, J. H.; van Hecke, M.; Somfai, E.; van Saarloos, W. Phys. Rev. 2004, E67, 030302(R)

(14) Henkes, S.; O’Hern, C. S.; Chakraborty, B. Phys. Rev. Lett. 2007, 99, 038002.

(15) (a) Liu, C.-H.; Nagel, S. R.; Schecter, D. A.; Coppersmith, S. N.; Majumdar, S.; Narayan, O.; Witten, T. A. Science 1995, 269, 513. (b) Meuth, D. M.; Jaeger, H. M.; Nagel, S. R. Phys. Rev. 1998, E 57, 3164. (c) Tsoungui, O.; Vallet, D.; Charmet, J. Granular Matter 1998, 1, 65.

(16) (a) Coppersmith, S. N.; Liu, C.-h.; Majumdar, S.; Narayan, O.; Witten, T. A. Phys. Rev. E 1996, 53, 4673-4685. (b) Radjai, F.; Wolf, D. E.; Jean, M.; Roux, S.; Moreau, J. In Powders and Grains 97; BehringerR. P., Jenkins, J. T. Eds.; Balkema: Rotterdam, 1997; pp 211. (c) Goldenberg, C.; Goldhirsch, I. Granular Matter 2003, 6, 87. (d) Snoeijer, J. H.; van Hecke, M.; Somfai, E.; van Saarloos, W. Phys. Rev. 2004, E 67, 030302(R).

(17) Blumenfeld, R.; Edwards, S. F.; Ball, R. C. J. Phys.: Cond. Mat. 2005, 17, S2481.

(18) Blumenfeld, R. IMA Volumes in Mathematics and its Applications. In Modeling of Soft Matter; Calderer, M-.C. T., Terentjev, E. M. Eds.; Springer-Verlag: New York 2005; Vol. 141.

(19) (a) Gerritsen, M.; Kreiss, G.; Blumenfeld, R. Phys. Rev. Lett. 2008, 101, 098001. (b) Gerritsen, M.; Kreiss, G.; Blumenfeld, R. Physica 2008, A 387, 6263.

(20) See, e.g. (a) Carrillo, J. A.; Cercignani, C.; Gamba, I. M. Phys. Rev. 2000, E 62, 7700. (b) Bisil, M.; Carrillo, J.; Toscani, G. J. Stat. Phys. $\mathbf{2 0 0 5}, 118,301$, and references therein.

(21) See, e.g. (a) Metzger, P. T. Phys. Rev. 2000, E 62, 7700. (b) Bisi1, M.; Carrillo, J.; Toscani, G. J. Stat. Phys. 2005, 118, 301, and references therein.

(22) Edwards, S. F. J. Stat. Phys. 2004, 116, 29.

(23) (a) Radjai; Roux Phys. Rev. Lett. 2002, 89, 064302; (b) Utter, B.; Behringer, R. P. Phys. Rev. 2004, E 69, 031308.

(24) (a) Brujic, J.; Edwards, S. F.; Hopkinson, I.; Makse, H. A. Physica 2003, A 327, 201. (b) Majmudar, T. S.; Behringer, R. P. Nature, London 2005, 435, 1079. (c) Zhou, J.; Long, S.; Wang, Q.; Dinsmore, A. D. Science 2006, 312, 1631

(25) (a) Sexton, M. G.; Socolar, J. E. S.; Schaeffer, D. G. Phys. Rev. E 1999, 60, 1999-2008. (b) van Eerd, A. R. T.; Ellenbroek, W. G.; van Hecke, M.; Snoeijer, J. H.; Vlugt, T.J. H. Phys. Rev. 2007, E 75, 060302R.

(26) Tighe, B. P.; van Eerd, A. R. T.; Vlugt, T. J. H. Phys. Rev. Lett. 2008, 100, 238001.

JP809768Y 